\title{
Bloqueo auriculoventricular avanzado inducido por ejercicio: síndrome vasovagal
}

Ricardo Pereira ${ }^{1,2^{*}}$, Geraldo Bezerra da Silva Junior ${ }^{2,3}$, Pedro Sérgio Cunha Costa ${ }^{1}$

1 Serviço de Cardiologia, Hospital São Mateus. Fortaleza, Ceará, Brasil.

2 Departamento de Medicina Clínica, Faculdade de Medicina, Universidade Federal do Ceará. Fortaleza, Ceará, Brasil.

3 Curso de Medicina, Centro de Ciências da Saúde, Universidade de Fortaleza. Fortaleza, Ceará, Brasil.

\section{Introducción:}

Bloqueo auriculoventricular y el síndrome vasovagal puede ocurrir en las personas jóvenes durante el peak del ejercicio y al inicio de la fase de recuperación ${ }^{1}$.

El síncope es la causa más frecuente de pérdida transitoria de la conciencia en humanos ${ }^{2,3}$.

La principal causa de pérdida de conciencia transitoria en pacientes jóvenes es el síncope vasovagal. Después de consentimiento del paciente, presentamos el caso de un paciente joven con síndrome vasovagal inducida por ejercicio.

\section{Caso clínico:}

Un hombre sedentario de 41 años fue referido a la prueba ergométrica para establecer un diagnóstico de dolor torácico atípico. El paciente no estaba tomando ningún tipo de medicamento, pero informó historia familiar de hipertensión y enfermedad coronaria. La prueba ergométrica se realizó de acuerdo con el protocolo de Bruce. Hallazgos electrocardiográficos fueron normales en reposo (Figura 1). La prueba fue interrumpida durante la etapa 4 , después de 10 minutos, debido al agotamiento físico. La presión arterial basal y al

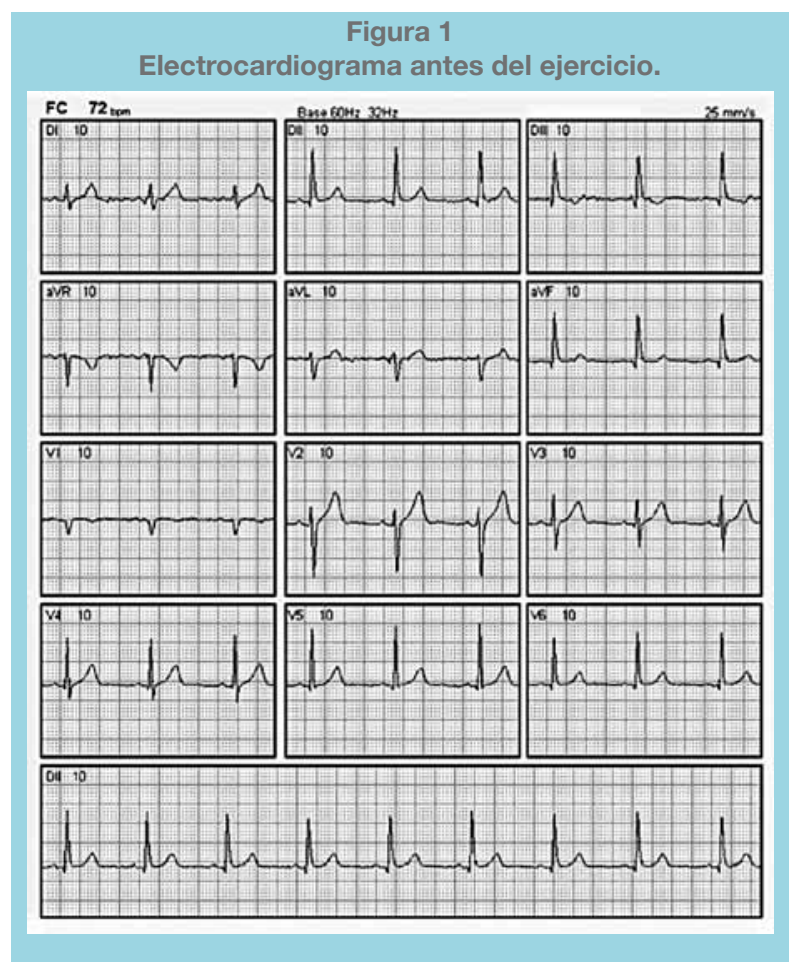

\section{Correspondencia:}

Prof. Dr. Ricardo Pereira Silva.

Rua República do Líbano,

630/1002. CEP 60160-140. Fortaleza, Ceará, Brasil.

Phone/Fax: +55 8532639001 / +55 8530643594 .

E-mail: ricardop@ufc.br,

geraldobezerrajr@yahoo.com.br 


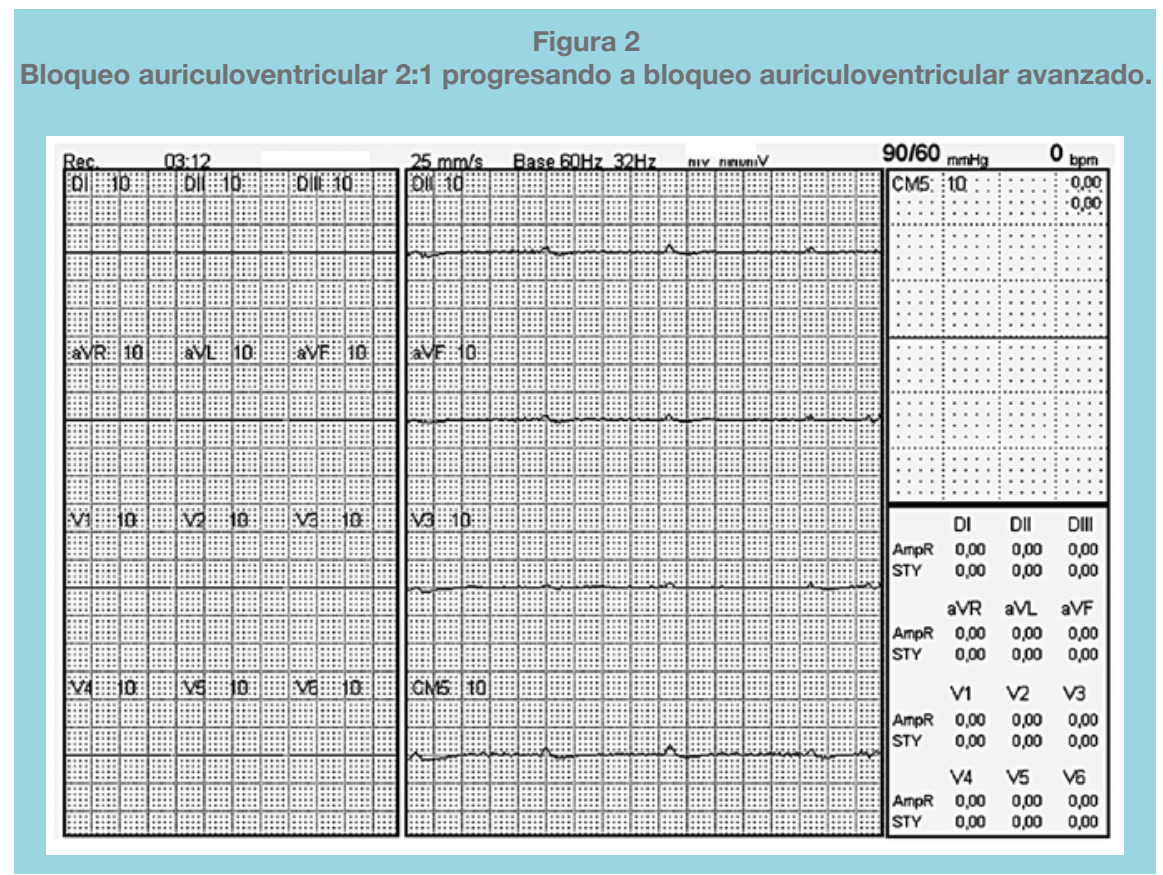

final del teste fueron $150 / 95 \mathrm{mmHg}$ y $170 / 80 \mathrm{mmHg}$, respectivamente. La frecuencia cardíaca $(\mathrm{FC})$ basal y final fue de $72 \mathrm{bpm}$ y $187 \mathrm{bpm}$, respectivamente, y el doble producto fue 31.790. Durante la recuperación, el paciente se quejó de dolor torácico atípico. El segmento ST se mantuvo inalterado a lo largo de la prueba. Después del segundo minuto de recuperación, el paciente informó que se iba a desmayar y le pidió ayuda. La presión arterial era 90/60 mmHg y la FC fue de 90 latidos por minuto. Cuando el paciente se colocó en decúbito dorsal y las extremidades inferiores se elevaron los síntomas persistieron, con acentuada palidez mucosa. El monitor mostró bradicardia en desarrollo hacia el bloqueo auriculoventricular 2:1. En este punto, el paciente fue orientado a provocar tos, para intentar normalizar la conducción auriculoventricular. Sin embargo, la bradicardia (FC media: 45 bpm) persistió y se convirtió en bloqueo auriculoventricular avanzado (Figura 2), pero la conducción se normalizó por la persistencia de tos. Sólo después de dos minutos el ritmo se hizo sinusoidal con bloqueo auriculoventricular de primer grado y FC normal (aproximadamente 90 latidos por minuto). Se inyectó inmediatamente solución salina a través de una vena periférica y la presión arterial se normalizó tras 20 minutos.

\section{Discusión:}

Este paciente presentó bradicardia sinusal en la fase de recuperación, bloqueo auriculoventricular 2:1 y bloqueo auriculoventricular de alto grado e hipoten- sión arterial, probablemente debido al reflejo vagal. Este fenómeno se observa en pacientes jóvenes sometidos a ejercicio exhaustivo y ocurre en el peak del ejercicio y al inicio de la fase de recuperación ${ }^{1}$.

En los seres humanos en posición vertical, la circulación cerebral es particularmente susceptible a la hipotensión arterial, debido al efecto de la gravedad que hace que la presión de perfusión cerebral sea $20 \mathrm{mmHg}$ más baja que en la arteria braquial ${ }^{2}$.

El síncope es la causa más frecuente de pérdida transitoria de la consciencia ${ }^{3}$. Hay cuatro modos de fallo de la circulación sistémica que puede ocasionar un síncope: 1) insuficiencia de la acción de bombeo del corazón, arritmia cardíaca y cardiopatía estructural, es decir, puede reducir el gasto cardíaco, causando síncope; 2) La presión arterial baja debido a la reducción del tono vascular y la falta de prevención de la acumulación de sangre en las piernas y en el abdomen al estar de pie, 3) el llenado insuficiente de la circulación, como ocurre en la hipovolemia, 4) hipotensión arterial sistémica como resultado de la vasodilatación refleja o bradicardia o ambas, mediada por los cambios en el tráfico neural aferente autonómica (síncope reflejo) ${ }^{4,5}$. La investigación diagnóstica de los pacientes con síncope relacionado con el ejercicio debe incluir la evaluación del riesgo de muerte cardíaca aguda. En los jóvenes, las miocardiopatías congénitas son la causa más común de muerte súbita cardíaca durante el esfuerzo físico. La miocardiopatía hipertrófica es la causa más importante y se ha estudiado en particular en 
los jóvenes atletas ${ }^{6}$.

Casi todos los episodios de pérdida transitoria de la conciencia en los jóvenes se deben a síncope reflejo. Un peak en la incidencia se observa alrededor de 15 años de edad y la incidencia acumulada durante toda la vida en mujeres adultas jóvenes es dos veces más alta que en hombres 5 .

Hay un reporte de caso de síncope durante el ejercicio, documentado con monitorización continua de la presión arterial durante la prueba ergométrica. Una mujer de 27 años de edad fue referida a la unidad de síncope después de un episodio de pérdida transitoria de la conciencia que ocurrió durante ejercicio aeróbico extenuante. Una reacción vasovagal inducido por el ejercicio fue la principal hipótesis y se documentó con monitorización continua de la presión arterial durante la prueba ergométrica. La paciente fue orientada a evitar el ejercicio máximo y terminación abrupta ${ }^{7}$.

Otro caso es reportado por Whyte et al. ${ }^{8}$. Una mujer de 25 años ha buscado el Centro Olímpico Británico de Medicina después de episodios de síncope y pré- síncope. Ella era una atleta de corrida internacional y solía correr 10 sesiones por semana. Fue sometida a una prueba de esfuerzo cardiopulmonar y al final del ejercicio se produjo una caída inmediata de la frecuencia cardíaca y presión arterial. La deportista fue diagnosticada con síndrome vasovagal inducido por ejercicio leve. La evidencia sugiere que el síncope relacionado con el ejercicio no está asociado con resultados adversos en los atletas de competición sin enfermedad cardíaca, y el atleta no debe ser excluido de la competición. Un programa de calentamiento para reducir el potencial para el desarrollo de síntomas vasovagales fue diseñado ${ }^{8}$. Hay algunas maniobras físicas para la prevención del síncope vasovagal. Estrategias de calentamiento, con tensión muscular, mejoran la hemodinámica de los pacientes con síncope vasovagal ${ }^{9}$.

En este caso clínico la maniobra que se utilizó para mejorar la conducción atrio-ventricular fue la tos, que se llevó a cabo de forma intermitente hasta que se fuera restablecido el ritmo sinusal.

\section{Referencias:}

1. ANDRADE J, BRITO FS, VILAS-BOAS F, CASTRO I, OLIVEIRA JA, GUIMARÃES JI, et al. II diretrizes da Sociedade Brasileira de Cardiologia sobre Teste Ergométrico. Arq Bras Cardiol 2002; 78: 1-18.

2. ROGER HAINSWORTH. Pathophysiology of syncope. Clin Auton Res 2004; 14: I18-I24.

3. KAUFMAN H, WIELING W. Syncope: a clinically guided diagnostic algorithm. Clin Auton Res 2004; 14: I87-I90.

4. THIJS RD, WIELING W, KAUFMAN H, VAN DIJK JG. Defining and classifying syncope. Clin Auton Res 2004; 14: I4-I8.

5. COLMAN N, NAHM K, GANZEBOOM KS, Shen WK, Reitsma JB, Linzer M, et al. Epidemiology of reflex syncope. Clin
Auton Res 2004; 14: I9-I17.

6. KREDIET CTP, WILDE AAM, WIELING W, HALLIWILL JR. Exercise related syncope, when it's not the heart. Clin Auton Res 2004; 14 : I25-I36.

7. KREDIET CTP, WILDE AAM, WIELING W, HALLIWILL JR. Syncope during exercise, documented with continuous blood pressure monitoring during ergometer testing. Clin Auton Res 2004; 15: I59-I62.

8. WHYTE G, STEPHENS N, BUDGETT R, SHARMA S, SHAVE RS, MCKENNA WJ. Exercise induced neurally mediated syncope in an elite rower: a treatment dilemma. Br J Sports Med 2004; 38:84-85.

9. KIM KH, CHO JG, LEE KO, SEO TJ, SHON CY, LIM SY, et al. Usefulness of physical maneuvers for prevention of vasovagal syncope. Circ J 2005; 69:1084-1088. 